\title{
A Call for Research: A Resource of Core Microbial Symbionts of the Arabidopsis thaliana Microbiome Ready and Awaiting Experimental Exploration
} $e-$ tra $^{*}$

\author{
Kenneth Dumack,,${ }^{1,2, \dagger}$ Melanie Sapp, ${ }^{3}$ Tiemo von Steimker, ${ }^{1,2}$ Anna Tatjana Mänz, ${ }^{1,2}$ \\ Laura Ellen Rose, ${ }^{3}$ and Michael Bonkowski ${ }^{1,2}$ \\ ${ }^{1}$ University of Cologne, Department of Terrestrial Ecology, Institute for Zoology, Zülpicher Str. \\ 47b, 50674 Köln, Germany \\ ${ }^{2}$ Cluster of Excellence on Plant Sciences (CEPLAS), University of Cologne, Institute of Zoology, \\ Terrestrial Ecology, Zülpicher Str 47b, 50674 Köln, Germany \\ ${ }^{3}$ Cluster of Excellence on Plant Sciences (CEPLAS), Heinrich Heine University, Population \\ Genetics, Universitätstrasse 1, 40225 Düsseldorf, Germany
}

\begin{abstract}
The characterization of specific subsets of soil microbiota in the rhizosphere and endosphere has led to the recognition of plant species-specific microbiomes. Most attention has been given to microbial prokaryotes and fungi. Only recently was convincing evidence for the existence of plant species-specific protist microbiomes presented. Although protists are expected to shape the composition of bacterial and fungal communities and, thereby, directly impact plant health, a lack of cultures of these important plant-symbiotic protists has hampered their experimental exploration. To facilitate empirical plant microbiome research, we sampled Arabidopsis thaliana, established 79 cultures covering nearly all major groups of plant-symbiotic Cercozoa (protists), and have made these publicly available. We discuss our findings and propose potential roles that these protists may have in structuring the plant microbiome.
\end{abstract}

Plants accommodate abundant and diverse communities of microorganisms (Bai et al. 2015; Lundberg et al. 2012). This unit, the so-called holobiont, is made up of specific microbial communities and their hosts (Rosenberg and Zilber-Rosenberg 2016). The establishment and structure of this holobiont are dependent on host-specific and environmental factors, including microbe-microbe interactions (Müller et al. 2016). A comprehensive overview of microbial interactions with regards to plant host was published recently (Hassani et al. 2018). Examples of microbial interactions shaping the plant holobiont include invasion by pathogens (Agler et al. 2016; Belhaj et al. 2017; Chapelle et al. 2016), cooperation (Kim et al. 2010; Mousa et al. 2016; Peterson et al. 2006), competition (Bernal et al. 2018; Raaijmakers and Mazzola 2012; Ryu 2015), control by phages (Bulgarelli et al. 2015; Morella et al. 2018), and predation (Benhamou et al. 2012; Rosenberg et al. 2009). Bacteria, especially, have been shown to affect plant growth and health but comparatively little is known about how these communities are affected by predation (Bordenstein and Theis 2015; Hassani et al. 2018; Xiong et al. 2020). Protists (unicellular eukaryotes) are the dominant group of bacterivores in the soil. They significantly impact the composition and function of rhizosphere bacterial communities and have been found to influence plant health (Bonkowski 2004; Trap et al. 2016; Xiong et al. 2020).

\footnotetext{
${ }^{\dagger}$ Corresponding author: K. Dumack, kenneth.dumack@uni-koeln.de

*The $e$-Xtra logo stands for "electronic extra" and indicates that supplementary materials are published online.
}

The author(s) declare no conflict of interest.

Accepted for publication 9 April 2021.
Funding

Support was provided by Deutsche Forschungsgemeinschaft (DFG, German Research Foundation), Germany's Excellence Strategy, grant number EXC-2048/1, project ID 390686111.

\section{Keywords}

plant health, plant parasites, predator-prey interactions, protist cultures, protists, rhizosphere 
Recent data revealed close affinities of a particular group of protists, the Cercozoa, to plants (Li et al. 2020; Santos et al. 2020; Simonin et al. 2020). Cercozoa-targeted studies further revealed rhizosphere- and phyllosphere-specific taxa (Dumack et al. 2020; Flues et al. 2018; Sapp et al. 2018). Ploch et al. (2016) and Sapp et al. (2018) characterized the protist microbiome of Arabidopsis thaliana. A significant finding was that the rhizosphere "core microbiome" (i.e., taxa that were consistently shared between several $A$. thaliana plants from different soils) was almost exclusively composed of the cercozoan class Sarcomonadea. The Sarcomonadea are considered the most abundant, genetically diverse, and widespread microbial bacterivores in soils, and their affinity to plants has been repeatedly documented (Fiore-Donno et al. 2018, 2019; Glücksman et al. 2010; Mahé et al. 2017; Oliverio et al. 2020). Almost all known Sarcomonadea species feed on bacteria (Braithwaite et al. 2018; Geisen et al. 2016; Ngo et al. 2018). Their predation patterns are species specific and evidence is mounting that grazing pressure exerted by these protists regulates the composition and function of bacterial communities (Flues et al. 2017; Glücksman et al. 2010; Xiong et al. 2020). As potent bacterivores, which can exert "top-down" regulatory effects on bacterial communities, Sarcomonadea may represent a heretofore unseen player shaping the plant microbiome (Xiong et al. 2020).

A necessary prerequisite to define the function of the plant microbiome is the execution of controlled experiments involving gnotobiotic hosts with different combinations of core microorganisms (Liu et al. 2019). The existing deficiency of cultures from microbiome protists has hampered such synthetic community (SynCom) approaches thus far. To rectify this situation, we have sampled $A$. thaliana from natural environments and cultured their root-associated communities of Sarcomonadea. We compare our results to those of the prior environmental sequencing study of Sapp et al. (2018) and discuss similarities and differences in our findings. To facilitate future microbiome research, we provide cultures for future reconstitution experiments.

\section{Resource Establishment}

Plants, including soil attached to roots, were collected in and around Cologne, Germany (Supplementary Table S1). Pieces of roots, approximately a centimeter in length, were picked, washed in WG medium (Bonkowski 2019), transferred to individual wells of a 24-well plate, and incubated for at least 1 day before investigation. The samples were incubated for up to 5 weeks and screened weekly for sarcomonadean cells with an inverted microscope (Nikon Eclipse TS100; Ph1; $\times 40$ to $\times 400$ magnification). Cells were picked with a glass micropipette and transferred into a new well of a 24-well plate with $1 \mathrm{ml}$ of WG medium each. The monoclonal cultures were subcultured approximately every 2 months. Isolated Viridiraptoridae species had to be cocultured with Spumella-like flagellates as eukaryotic prey.

We chose a barcoding approach to identify the taxa. We used the Cercozoa-specific primers and protocol described by Sapp et al. (2018) and published by Fiore-Donno et al. (2018). Sequences were aligned with the operational taxonomic units (OTUs) reported from Sapp et al. (2018) via the mafft linsi algorithm (Katoh and Standley 2013) and were considered to be identical if they showed $>97 \%$ similarity, the same cut-off used by Sapp et al. (2018) to define OTUs. We submitted the obtained sequences to the NCBI database and the established cultures, with duplicates removed, to the Culture Collection of Algae and Protozoa (CCAP) (https://www.ccap. ac.uk/); accession numbers are given in Supplementary Table S1.

Culture-based approaches are laborious and rarely capture the microbial diversity completely. However, cultures allow future in-depth experiments such as community reconstitution (Liu et al. 2019). Similar to the environmental analyses of Sapp et al. (2018), our culture-based approach revealed numerous sarcomonadean taxa in the rhizosphere of $A$. thaliana. Both methods resulted in a broad diversity of Cercomonadidae, Paracercomonadidae, Sandonidae, Allapsidae, and a single Viridiraptoridae OTU (Fig. 1). In Sapp et al. (2018), A. thaliana plants grown in distinct soil types shared several Paracercomonadidae OTUs, which led us to consider these consistently shared taxa as "core microbiome taxa". Our culture-based approach resulted in a similar picture and we were able to culture two of the four reported core microbiome Paracercomonadidae (e.g., strains C8 and C41). The sole Viridiraptoridae culture we provide (i.e., strain C35) is identical to the single Viridiraptoridae core microbiome taxon reported by Sapp et al. (2018) and, therefore, also falls under our definition of core microbiome taxa. From the numerous Sandonidae cultures we obtained, only one culture (C21) was identical to an OTU reported by Sapp et al. (2018). However, this culture, identical to OTU18 in Sapp et al. (2018), did not fall under our definition of the core microbiome because it showed no strong affiliation to the roots of $A$. thaliana in Sapp et al. (2018). 


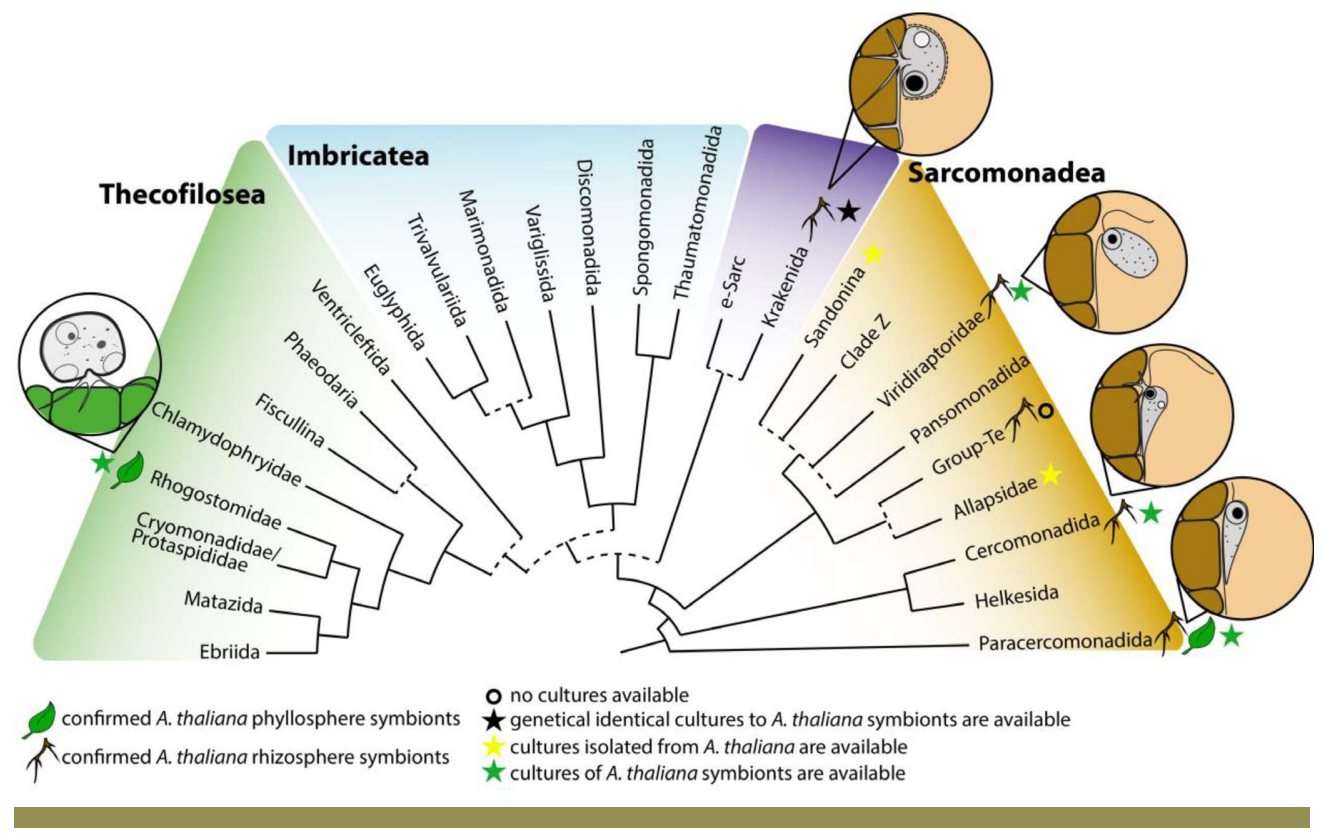

Fig. 1. Overview of cercozoan diversity and symbionts of Arabidopsis thaliana. Following a conservative approach, we only considered taxa that were repeatedly shown to be shared among several plants. All Sarcomonadea cultures that are currently publicly available result from this study. For cultures of non-sarcomonadean $A$. thaliana symbionts (i.e., the naked amoebae Krakenida and shelled amoebae Rhogostomidae), see Dumack et al. $(2016,2017)$. Drawings are not to scale.

In total, we were able to establish 79 cultures of 35 unique genotypes. Of these 35 unique protist cultures, 34 were stable over the entire length of the study. These 34 cultures are available upon request from the authors or from the CCAP (Supplementary Table S1). In total, we provide 8 genetically distinct cultures of the family Paracercomonadidae, 7 of Cercomonadidae, 10 of Allapsidae, 8 of Sandonidae, and 1 culture of Viridiraptoridae. We especially recommend the three core microbiome taxa (cultures C8, C41, and C35) and, potentially, C21 to be included in future experiments. Although taxon C21 was not classified as a core microbial taxon, it represents a phylogenetic clade distinct from the other cultures. The other established cultures may be used to complement such experiments; however, it is still not known whether these protists are merely common soil- or plant-associated protistan taxa or genuinely associated with $A$. thaliana.

\section{Perspective}

Soils accommodate a diverse assemblage of distantly related protistan taxa that fulfill complementary or redundant functions (Bates et al. 2012; Oliverio et al. 2020). From this pool, plant species recruit specific microorganisms via growth-limiting carbohydrates and distinct metabolite profiles provided in root exudates (Baetz and Martinoia 2014; Jones et al. 2009; Sasse et al. 2018; van Dam and Bouwmeester 2016). The plant-associated microbial community is strongly influenced by the site of collection and soil type, which presents different physical and chemical properties (Girvan et al. 2003; Sessitsch et al. 2001; Simonin et al. 2020; Ulrich and Becker 2006; Zhao et al. 2019, 2020). In addition to the variation that is caused by the soils the plants are growing in, there is evidence that a higher percentage of the variation in the plantassociated and nonassociated protist community composition is explained by the plants themselves (de Araujo et al. 2018; Dumack et al. 2020; Leff et al. 2018; Sapp et al. 2018; Simonin et al. 2020).

The plant host relies on microbial taxa to fulfill certain host-beneficial functions. Although numerous studies aimed to unravel the functional roles of plant-associated protists, often, readily available cultures were used for experiments; however, unfortunately, these cultures were not necessarily good representatives of genuine soil- or root-associated species. A typical example is the widespread and continuous usage of Tetrahymena spp. in soil experiments, although Tetrahymena spp. are freshwater ciliates that are absent in soils (Foissner 1998).

The here-provided cultures represent a diverse assemblage of genuine soil protists sampled from plants. The Sarcomonadea are mainly bacterivorous and a recent study determined 
them as key predictors of plant health, indicating that Sarcomonadea control plant-pathogenic bacteria by their species-specific prey preferences (Xiong et al. 2020). Thus, the here-provided cultures enable comprehensive feeding trials with bacterial cultures or in situ holobionts, thereby complementing our knowledge gathered from tests using model organisms such as Acanthamoeba castellanii (Amacker et al. 2020; Asiloglu et al. 2020; Gao et al. 2019; Murase et al. 2006; Rosenberg et al. 2009).

In general, the functions of plant-associated protists of Arabidopsis thaliana may extend beyond bacterivory (Dumack et al. 2019). One particular culture provided by us is an undescribed species of the Viridiraptoridae, which was found to be a core microbial symbiont of A. thaliana by Sapp et al. (2018). The few known species of the Viridiraptoridae (Fig. 1) are limnic and show parasite-like predation on filamentous algae (Hess and Melkonian 2013). The terrestrial species reported here is still undescribed and its prey spectrum is unexplored, although we were able to culture it with Spumella-like flagellates as eukaryotic prey. However, our own unpublished results indicate that terrestrial Viridiraptoridae can consume a variety of dead eukaryotic prey, including nematodes and plant cells. Whether this particular Viridiraptoridae isolate can infect living plant tissue is of considerable interest and is currently being tested. Preliminary data suggest a direct effect on plant fitness but further experiments are required to comprehensively understand the interaction between this isolate and $A$. thaliana. The remaining plant-associated sarcomonadean clade, Group Te (Fig. 1), could not be cultured by us, although it was found as core microbial symbionts by Sapp et al. (2018). The fact that we could not isolate it may hint at other requirements in culturing. It is unknown whether this group does not prey on bacteria or other microbial eukaryotes or whether it may have evaded our culturing approach because it is adapted to more extreme conditions such as oxygen-depleted environments that may occur in specific soil conditions (Howe et al. 2009, 2011).

\section{Conclusion}

The cultures provided here fill a vital gap in this field and facilitate experiments with gnotobiotic hosts similar to the commonly applied SynCom approach (Liu et al. 2019). We were able to provide cultures of all but one of the major Sarcomonadea lineages that were also found by Sapp et al. (2018).

\section{Literature Cited}

Agler, M. T., Ruhe, J., Kroll, S., Morhenn, C., Kim, S. T., Weigel, D., and Kemen, E. M. 2016. Microbial hub taxa link host and abiotic factors to plant microbiome variation. PLoS Biol. 14:e1002352.

Amacker, N., Gao, Z., Agaras, B. C., Latz, E., Kowalchuk, G. A., Valverde, C. F., Jousset, A., and Weidner, S. 2020. Biocontrol traits correlate with resistance to predation by protists in soil pseudomonads. Front. Microbiol. 11:3164.

Asiloglu, R., Shiroishi, K., Suzuki, K., Can, O., and Murase, J. 2020. Protistenhanced survival of a plant growth promoting rhizobacteria, Azospirillum sp. B510, and the growth of rice (Oryza sativa L.) plants. Appl. Soil Ecol. 154:103599.

Baetz, U., and Martinoia, E. 2014. Root exudates: The hidden part of plant defense. Trends Plant Sci. 19:90-98.

Bai, Y., Müller, D. B., Srinivas, G., Garrido-Oter, R., Potthoff, E., Rott, M., Dombrowski, N., Münch, P. C., Spaepen, S., Remus-Emsermann, M., Hüttel, B., McHardy, A. C., and Vorholt, J. A. 2015. Functional overlap of the Arabidopsis leaf and root microbiota. Nature 528:364-369.

Bates, S. T., Clemente, J. C., Flores, G. E., Walters, W. A., Parfrey, L. W., Knight, R., and Fierer, N. 2012. Global biogeography of highly diverse protistan communities in soil. ISME J. 7:652-659.

Belhaj, K., Cano, L. M., Prince, D. C., Kemen, A., Yoshida, K., Dagdas, Y. F., Etherington, G. J., Schoonbeek, H. J., van Esse, H. P., Jones, J. D. G., Kamoun, S., and Schornack, S. 2017. Arabidopsis late blight: Infection of a nonhost plant by Albugo laibachii enables full colonization by Phytophthora infestans. Cell. Microbiol. 19:e12628.

Benhamou, N., le Floch, G., Vallance, J., Gerbore, J., Grizard, D., and Rey, P. 2012. Pythium oligandrum: An example of opportunistic success. Microbiology 158:2679-2694.

Bernal, P., Llamas, M. A., and Filloux, A. 2018. Type VI secretion systems in plantassociated bacteria. Environ. Microbiol. 20:1-15.
Bonkowski, M. 2004. Protozoa and plant growth: The microbial loop in soil revisited. New Phytol. 162:617-631.

Bonkowski, M. 2019. Microcosm approaches to investigate multitrophic interactions between microbial communities in the rhizosphere of plants. Pages 255-270 in: Methods in Rhizosphere Biology Research. D. Reinhardt and A. K. Sharma, eds. Springer, Singapore.

Bordenstein, S. R., and Theis, K. R. 2015. Host biology in light of the microbiome: Ten principles of holobionts and hologenomes. PLoS Biol. 13:e1002226.

Braithwaite, K. S., Ngo, C. N., and Croft, B. J. 2018. Confirmation that the novel Cercozoa Phytocercomonas venanatans is the cause of the disease chlorotic streak in sugarcane. Phytopathology 108:487-494.

Bulgarelli, D., Garrido-Oter, R., Münch, P. C., Weiman, A., Dröge, J., Pan, Y., McHardy, A. C., and Schulze-Lefert, P. 2015. Structure and function of the bacterial root microbiota in wild and domesticated barley. Cell Host Microbe 17:392-403.

Chapelle, E., Mendes, R., Bakker, P. A. H., and Raaijmakers, J. M. 2016. Fungal invasion of the rhizosphere microbiome. ISME J. 10:265-268.

de Araujo, A. S. F., Mendes, L. W., Lemos, L. N., Antunes, J. E. L., Beserra, J. E. A., Jr., de Lyra, M. d. C. C. P., Figueiredo, M. d. V. B., de Almeida Lopes, A. C., Gomes, R. L. F., Bezerra, W. M., Melo, V. M. M., de Araujo, F. F.., and Geisen, S. 2018. Protist species richness and soil microbiome complexity increase towards climax vegetation in the Brazilian Cerrado. Commun. Biol. 1:135.

Dumack, K., Feng, K., Flues, S., Sapp, M., Schreiter, S., Grosch, R., Rose, L., Deng, Y., Smalla, K., and Bonkowski, M., 2020. What drives the assembly of plant-associated protist microbiomes? bioRxiv. https://doi.org/10.1101/2020.02. 16.951384

Dumack, K., Fiore-Donno, A. M., Bass, D., and Bonkowski, M. 2019. Making sense of environmental sequencing data: Ecologically important functional traits of the protistan groups Cercozoa and Endomyxa (Rhizaria). Mol. Ecol. Resour. 20:398-403.

Dumack, K., Flues, S., Hermanns, K., and Bonkowski, M. 2017. Rhogostomidae (Cercozoa) from soils, roots and plant leaves (Arabidopsis thaliana): Description 
of Rhogostoma epiphylla sp. nov. and R. cylindrica sp. nov. Eur. J. Protistol. 60:76-86.

Dumack, K., Schuster, J., Bass, D., and Bonkowski, M. 2016. A novel lineage of 'naked filose amoebae'; Kraken carinae gen. nov. sp. nov. (Cercozoa) with a remarkable locomotion by disassembly of its cell body. Protist 167:268-278.

Fiore-Donno, A. M., Richter-Heitmann, T., Degrune, F., Dumack, K., Regan, K. M., Marhan, S., Boeddinghaus, R. S., Rillig, M. C., Friedrich, M. W., Kandeler, E., and Bonkowski, M. 2019. Functional traits and spatio-temporal structure of a major group of soil protists (Rhizaria: Cercozoa) in a temperate grassland. Front. Microbiol. 10:1332.

Fiore-Donno, A. M., Rixen, C., Rippin, M., Glaser, K., Samolov, E., Karsten, U., Becker, B., and Bonkowski, M. 2018. New barcoded primers for efficient retrieval of cercozoan sequences in high-throughput environmental diversity surveys, with emphasis on worldwide biological soil crusts. Mol. Ecol. Resour. 18:229-239.

Flues, S., Bass, D., and Bonkowski, M. 2017. Grazing of leaf-associated Cercomonads (Protists: Rhizaria: Cercozoa) structures bacterial community composition and function. Environ. Microbiol. 19:3297-3309.

Flues, S., Blokker, M., Dumack, K., and Bonkowski, M. 2018. Diversity of Cercomonad species in the phyllosphere and rhizosphere of different plant species with a description of Neocercomonas epiphylla (Cercozoa, Rhizaria) a leaf-associated protist. J. Eukaryot. Microbiol. 65:587-599.

Foissner, W. 1998. An updated compilation of world soil ciliates (Protozoa, Ciliophora), with ecological notes, new records, and descriptions of new species. Eur. J. Protistol. 34:195-235.

Gao, Z., Karlsson, I., Geisen, S., Kowalchuk, G., and Jousset, A. 2019. Protists: Puppet masters of the rhizosphere microbiome. Trends Plant Sci. 24:165-176.

Geisen, S., Koller, R., Hünninghaus, M., Dumack, K., Urich, T., and Bonkowski, M. 2016. The soil food web revisited: Diverse and widespread mycophagous soil protists. Soil Biol. Biochem. 94:10-18.

Girvan, M. S., Bullimore, J., Pretty, J. N., Osborn, A. M., and Ball, A. S. 2003. Soil type is the primary determinant of the composition of the total and active bacterial communities in arable soils. Appl. Environ. Microbiol. 69:1800-1809.

Glücksman, E., Bell, T., Griffiths, R. I., and Bass, D. 2010. Closely related protist strains have different grazing impacts on natural bacterial communities. Environ. Microbiol. 12:3105-3113.

Hassani, M. A., Durán, P., and Hacquard, S. 2018. Microbial interactions within the plant holobiont. Microbiome 6:58.

Hess, S., and Melkonian, M. 2013. The mystery of clade X: Orciraptor gen. nov. and Viridiraptor gen. nov. are highly specialised, algivorous amoeboflagellates (Glissomonadida, Cercozoa). Protist 164:706-747.

Howe, A. T., Bass, D., Chao, E. E., and Cavalier-Smith, T. 2011. New genera, species, and improved phylogeny of Glissomonadida (Cercozoa). Protist 162:710-722.

Howe, A. T., Bass, D., Vickerman, K., Chao, E. E., and Cavalier-Smith, T. 2009. Phylogeny, taxonomy, and astounding genetic diversity of Glissomonadida ord. nov., the dominant gliding zooflagellates in soil (Protozoa: Cercozoa). Protist 160:159-189.

Jones, D. L., Nguyen, C., and Finlay, R. D. 2009. Carbon flow in the rhizosphere: Carbon trading at the soil-root interface. Plant Soil 321:5-33.

Katoh, K., and Standley, D. M. 2013. MAFFT Multiple Sequence Alignment Software Version 7: Improvements in Performance and Usability. Mol, Biol. Evol. 30:772-780.

Kim, K., Yim, W., Trivedi, P., Madhaiyan, M., Boruah, H. P. D., Islam, M. R., Lee, G., and Sa, T. 2010. Synergistic effects of inoculating arbuscular mycorrhizal fungi and Methylobacterium oryzae strains on growth and nutrient uptake of red pepper (Capsicum annuum L.). Plant Soil 327:429-440.

Leff, J. W., Bardgett, R. D., Wilkinson, A., Jackson, B. G., Pritchard, W. J., De Long, J. R., Oakley, S., Mason, K. E., Ostle, N. J., Johnson, D., Baggs, E. M., and Fierer, N. 2018. Predicting the structure of soil communities from plant community taxonomy, phylogeny, and traits. ISME J. 12:1794-1805.

Li, J., Mavrodi, O. V., Hou, J., Blackmon, C., Babiker, E. M., and Mavrodi, D. V. 2020. Comparative analysis of rhizosphere microbiomes of Southern Highbush blueberry (Vaccinium corymbosum L.), Darrow's blueberry (V. darrowii Camp), and Rabbiteye blueberry (V. virgatum Aiton). Front. Microbiol. 11:370.

Liu, Y. X., Qin, Y., and Bai, Y. 2019. Reductionist synthetic community approaches in root microbiome research. Curr. Opin. Microbiol. 49:97-102.

Lundberg, D. S., Lebeis, S. L., Paredes, S. H., Yourstone, S., Gehring, J., Malfatti, S., Tremblay, J., Engelbrektson, A., Kunin, V., Glavina, T., Edgar, R. C., Eickhorst, T., and Ley, R. E. 2012. Defining the core Arabidopsis thaliana root microbiome. Nature 488:86-90.

Mahé, F., de Vargas, C., Bass, D., Czech, L., Stamatakis, A., Lara, E., Singer, D., Mayor, J., Bunge, J., Sernaker, S., Siemensmeyer, T., Trautmann, I., Romac, S., Berney, C., Kozlov, A., Mitchell, E. A. D., Seppey, C. V. W., Egge, E., Lentendu,
G., Wirth, R., Trueba, G., and Dunthorn, M. 2017. Parasites dominate hyperdiverse soil protist communities in Neotropical rainforests. Nat. Ecol. Evol. 1:0091.

Morella, N. M., Gomez, A. L., Wang, G., Leung, M. S., and Koskella, B. 2018. The impact of bacteriophages on phyllosphere bacterial abundance and composition. Mol. Ecol. 27:2025-2038.

Mousa, W. K., Shearer, C., Limay-Rios, V., Ettinger, C. L., Eisen, J. A., and Raizada, M. N. 2016. Root-hair endophyte stacking in finger millet creates a physicochemical barrier to trap the fungal pathogen Fusarium graminearum. Nat. Microbiol. 1:16167.

Müller, D. B., Vogel, C., Bai, Y., and Vorholt, J. A. 2016. The plant microbiota: Systems-level insights and perspectives. Annu. Rev. Genet. 50:211-234.

Murase, J., Noll, M., and Frenzel, P. 2006. Impact of protists on the activity and structure of the bacterial community in a rice field soil. Appl. Environ. Microbiol. 72:5436-5444.

Ngo, C. N., Braithwaite, K. S., Bass, D., Young, A. J., and Croft, B. J. 2018. Phytocercomonas venanatans, a new species of Cercozoa associated with chlorotic streak of sugarcane. Phytopathology 108:479-486

Oliverio, A. M., Geisen, S., Delgado-Baquerizo, M., Maestre, F. T., Turner, B. L., and Fierer, N. 2020. The global-scale distributions of soil protists and their contributions to belowground systems. Sci. Adv. 6:eaax8787.

Peterson, S. B., Dunn, A. K., Klimowicz, A. K., and Handelsman, J. 2006. Peptidoglycan from Bacillus cereus mediates commensalism with rhizosphere bacteria from the Cytophaga-Flavobacterium group. Appl. Environ. Microbiol. 72:5421-5427.

Ploch, S., Rose, L. E., Bass, D., and Bonkowski, M. 2016. High diversity revealed in leaf-associated protists (Rhizaria: Cercozoa) of Brassicaceae. J. Eukaryot. Microbiol. 63:635-641.

Raaijmakers, J. M., and Mazzola, M. 2012. Diversity and natural functions of antibiotics produced by beneficial and plant pathogenic bacteria. Annu. Rev. Phytopathol. 50:403-424.

Rosenberg, E., and Zilber-Rosenberg, I. 2016. Microbes drive evolution of animals and plants: The hologenome concept. mBio 7:e01395-15.

Rosenberg, K., Bertaux, J., Krome, K., Hartmann, A., Scheu, S., and Bonkowski, M. 2009. Soil amoebae rapidly change bacterial community composition in the rhizosphere of Arabidopsis thaliana. ISME J. 3:675-684.

Ryu, C. M. 2015. Against friend and foe: Type 6 effectors in plant-associated bacteria. J. Microbiol. 53:201-208.

Santos, S. S., Schöler, A., Nielsen, T. K., Hansen, L. H., Schloter, M., and Winding, A. 2020. Land use as a driver for protist community structure in soils under agricultural use across Europe. Sci. Total Environ. 717:137228.

Sapp, M., Ploch, S., Fiore-Donno, A. M., Bonkowski, M., and Rose, L. E. 2018. Protists are an integral part of the Arabidopsis thaliana microbiome. Environ. Microbiol. 20:30-43.

Sasse, J., Martinoia, E., and Northen, T. 2018. Feed your friends: Do plant exudates shape the root microbiome? Trends Plant Sci. 23:25-41.

Sessitsch, A., Weilharter, A., Gerzabek, M. H., Kirchmann, H., and Kandeler, E. 2001. Microbial population structures in soil particle size fractions of a long-term fertilizer field experiment. Appl. Environ. Microbiol. 67:4215-4224.

Simonin, M., Dasilva, C., Terzi, V., Ngonkeu, E. L. M., Diouf, D., Kane, A., Béna, G., and Moulin, L. 2020. Influence of plant genotype and soil on the wheat rhizosphere microbiome: Evidences for a core microbiome across eight African and European soils. FEMS Microbiol. Ecol. 96:fiaa067.

Trap, J., Bonkowski, M., Plassard, C., Villenave, C., and Blanchart, E. 2016. Ecological importance of soil bacterivores for ecosystem functions. Plant Soil 398:1-24.

Ulrich, A., and Becker, R. 2006. Soil parent material is a key determinant of the bacterial community structure in arable soils. FEMS Microbiol. Ecol. 56:430-443.

van Dam, N. M., and Bouwmeester, H. J. 2016. Metabolomics in the rhizosphere: Tapping into belowground chemical communication. Trends Plant Sci. 21:256-265.

Xiong, W., Song, Y., Yang, K., Gu, Y., Wei, Z., Kowalchuk, G. A., Xu, Y., Jousset, A., Shen, Q., and Geisen, S. 2020. Rhizosphere protists are key determinants of plant health. Microbiome 8:27.

Zhao, Z. B., He, J. Z., Geisen, S., Han, L. L., Wang, J. T., Shen, J. P., Wei, W. X., Fang, Y. T., Li, P. P., and Zhang, L. M. 2019. Protist communities are more sensitive to nitrogen fertilization than other microorganisms in diverse agricultural soils. Microbiome 7:33.

Zhao, Z. B., He, J. Z., Quan, Z., Wu, C. F., Sheng, R., Zhang, L. M., and Geisen, S. 2020. Fertilization changes soil microbiome functioning, especially phagotrophic protists. Soil Biol. Biochem. 148:107863. 